\title{
Physical Properties of Superconducting $\mathrm{V}_{0.98}{ }^{57} \mathrm{Fe}_{0.02}$ Alloy Studied by TMS and SQUID Magnetometer
}

\author{
R. IDCZAK ${ }^{* \dagger}$ AND V.H. TRAN \\ Institute of Low Temperature and Structure Research, Polish Academy of Sciences, \\ P.O. Box 1410, 50-422 Wrocław, Poland
}

\begin{abstract}
A sample of vanadium with 2 at.\% of ${ }^{57} \mathrm{Fe}$ was prepared by the arc-melting technique, annealed in a vacuum at $1270 \mathrm{~K}$ for $2 \mathrm{~h}$ and measured by ${ }^{57} \mathrm{Fe}$ transmission Mössbauer spectroscopy in the temperature range $1.4 \mathrm{~K}-300 \mathrm{~K}$ as well as using SQUID magnetometer in the temperature range $1.8 \mathrm{~K}-300 \mathrm{~K}$. The magnetic data showed that the $\mathrm{V}_{0.98}{ }^{57} \mathrm{Fe}_{0.02}$ alloy is a type-II superconductor with transition temperature $T_{\mathrm{c}}=3.7 \mathrm{~K}$ and upper critical magnetic field $H_{c 2}$ close to $4.8 \mathrm{kOe}$ at $1.8 \mathrm{~K}$. The observed Mössbauer spectra consist of one doublet with small quadrupole splitting over the whole temperature range studied. The weighted average of the two Debye temperature values from temperature dependence of the isomer shift and the total spectral was estimated to be approximately $602(90) \mathrm{K}$ for ${ }^{57} \mathrm{Fe}$. Abnormal behaviour of the hyperfine parameters at or near $T_{\mathrm{c}}$ was not observed.
\end{abstract}

DOI: 10.12693/APhysPolA.134.1066

PACS/topics: electronic structure of bulk materials, superconductivity, electronic structure calculations

\section{Introduction}

Pure vanadium is a type-II conventional superconductor with transition temperature $T_{\mathrm{c}}=5.4 \mathrm{~K}$ and upper critical magnetic field $H_{c 2}$ close to $3 \mathrm{kOe}[1-3]$. The substitution of vanadium by $d$-electron transition metals, such as $\mathrm{Fe}, \mathrm{Co}, \mathrm{Ni}$, and $\mathrm{Cr}$ causes a gradual decrease in superconducting transition temperature $T_{\mathrm{c}}$. In the case of substitutional bcc $\mathrm{V}_{1-x} \mathrm{Fe}_{x}$ alloys, this effect is mainly connected with the change in lattice and electronic parameters, e.g., density of states at Fermi level [4]. The magnetic effects from $\mathrm{Fe}$ on superconducting properties of the $\mathrm{V}_{1-x} \mathrm{Fe}_{x}$ alloys can be eluded due to fact that the Fe magnetic moment vanishes when the number of nearest neighbours $\mathrm{V}$ atoms is larger than 7 [5].

In this work, a Quantum-Design superconducting quantum interference device (SQUID) magnetometer and the ${ }^{57} \mathrm{Fe}$ transmission Mössbauer spectroscopy (TMS) were used to study physical properties of $\mathrm{V}_{0.98}{ }^{57} \mathrm{Fe}_{0.02}$ alloy in the temperature range $1.5 \mathrm{~K}-300 \mathrm{~K}$. The combination of these two experimental methods could provide interesting information about the low-temperature magnetic properties of iron atoms dissolved in vanadium matrix as well as allows one to estimate important parameters such as the Debye temperature $\Theta_{\mathrm{D}}$ and electronphonon coupling $\bar{\lambda}_{\text {el-ph }}$ in the studied material. To the best of our knowledge, this work is the first ever-reported low-temperature study on $\Theta_{\mathrm{D}}$ and $\bar{\lambda}_{\mathrm{el}-\mathrm{ph}}$ in substitutional $\mathrm{V}_{1-x} \mathrm{Fe}_{x}$ alloys. Here, it should be recalled that $\Theta_{\mathrm{D}}$ and $\bar{\lambda}_{\mathrm{el}-\mathrm{ph}}$ values for various $\mathrm{V}-\mathrm{Fe}$ alloys were reported in Ref. [4], where the $\Theta_{\mathrm{D}}$ values were calculated under the assumption that $\Theta_{\mathrm{D}}$ varies linearly between $\Theta_{\mathrm{D}}$ values for pure $\mathrm{V}$ and $\mathrm{Fe}$.

\footnotetext{
*corresponding author; e-mail: r.idczak@intibs.pl

†on leave from Institute of Experimental Physics, University of Wrocław, Poland
}

\section{Experimental details}

The sample of $\mathrm{V}_{0.98}{ }^{57} \mathrm{Fe}_{0.02}$ was prepared by the arcmelting technique. Appropriate amounts of the pure ${ }^{57} \mathrm{Fe}$ isotope $(99.9 \%)$ and $\mathrm{V}(99.8 \%)$ were melted in a watercooled copper crucible under a Ti-gettered purified argon atmosphere. Melting procedure was repeated twice to ensure homogeneity. It was found that the measured weight loss after the melting process was below $0.2 \%$ of the original weight, thus the chemical composition of the obtained ingot is very close to the nominal one. Resulting alloy was cold-rolled to the final thickness of about $0.035 \mathrm{~mm}$. After the cold-rolling process, to obtain homogeneous and defect-free sample, the foil was annealed in the vacuum furnace at $1270 \mathrm{~K}$ for $2 \mathrm{~h}$ and then slowly cooled to room temperature during $6 \mathrm{~h}[6]$. The base pressure during the annealing procedure was lower than $10^{-4} \mathrm{~Pa}$.

The dc magnetization measurements from $1.8 \mathrm{~K}$ to $300 \mathrm{~K}$ in magnetic fields up to $10 \mathrm{kOe}$ were performed using a SQUID magnetometer (Quantum Design MPMS XL-7). The presented values of the magnetization and magnetic susceptibility have an uncertainty of less than $5 \%$.

The ${ }^{57} \mathrm{Fe}$ Mössbauer spectra were recorded in transmission geometry with a conventional constant-acceleration spectrometer, using a ${ }^{57} \mathrm{Co}$-in-Rh standard source with a full width at half maximum (FWHM) of $0.22 \mathrm{~mm} / \mathrm{s}$. Sample temperature in the range $1.4 \mathrm{~K}-300 \mathrm{~K}$ was controlled using a variable-temperature insert in an Oxford Instruments Spectromag cryostat. The obtained TMS spectra were analysed using a least-squares fitting procedure which allows to determine the hyperfine interactions parameters such as isomer shift $(I S)$ and quadrupole splitting $(Q S)$ as well as the absorption spectral areas $(C)$. All the $I S$ values presented in this paper are related to the $\alpha$-Fe standard at room temperature. 


\section{Results and discussion}

Figure 1 shows the temperature dependence of internal susceptibility $\chi_{\text {int }}$ (in SI unit) of the $\mathrm{V}_{0.98}{ }^{57} \mathrm{Fe}_{0.02}$ alloy in several magnetic fields. The $\chi_{\text {int }}$ values were estimated taking into account demagnetization factor $N \approx 1$ (a thin plate in a perpendicular field) and the relation $\chi_{\text {int }}=\chi_{\text {ext }} /\left(1-N \chi_{\text {ext }}\right)$, where $\chi_{\text {ext }}$ is the measured external susceptibility $\chi_{\text {ext }}$. As one can see, the measurements performed in 10 Oe and 20 Oe reveal the onset of diamagnetic signal at $3.7 \mathrm{~K}$, while $\chi_{\text {int }}$ values obtained in a field of $10 \mathrm{kOe}$, remains positive and almost unchanged even at $2 \mathrm{~K}$. This result is in good agreement with data presented in work [4], where the superconducting transition temperature $T_{\mathrm{c}}$ for $\mathrm{V}_{0.9811} \mathrm{Fe}_{0.0189}$ alloy was determined to amount to $3.96 \mathrm{~K}$ and the upper critical magnetic field $H_{c 2}(0)=6.72 \mathrm{kOe}$. Finally, it is worth noting that the internal susceptibility $\chi_{\text {int }}$ of the studied sample at $1.8 \mathrm{~K}$ in $10 \mathrm{Oe}$ is equal to -0.89 , corresponding to superconducting volume fraction of $89 \%$.

Figure 2a presents the measured magnetization $M(T)$ of the $\mathrm{V}_{0.98}{ }^{57} \mathrm{Fe}_{0.02}$ alloy at $10 \mathrm{kOe}$ as a function of

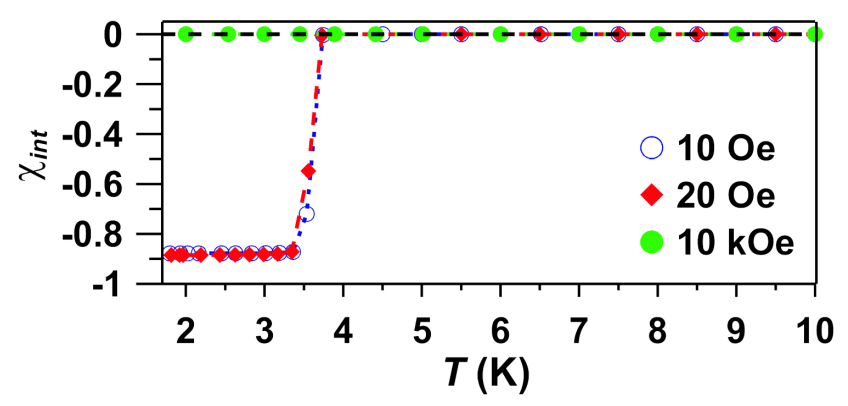

Fig. 1. Temperature dependence of internal susceptibility of $\mathrm{V}_{0.98}{ }^{57} \mathrm{Fe}_{0.02}$ in several magnetic fields.
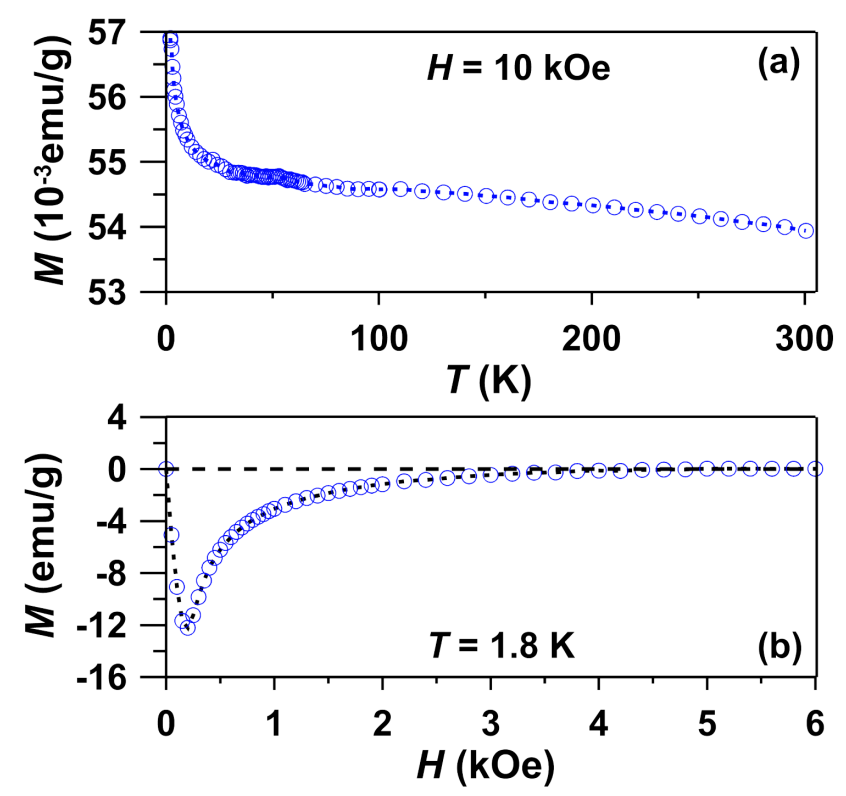

Fig. 2. (a) The temperature dependence of the magnetization in a field of $10 \mathrm{kOe}$, (b) magnetization versus applied fields at $1.8 \mathrm{~K}$ for the $\mathrm{V}_{0.98}{ }^{57} \mathrm{Fe}_{0.02}$ sample. temperature. As one can see, magnetization above $25 \mathrm{~K}$ is nearly temperature-independent, implying substantially Pauli paramagnetism of the conduction electrons. Below $25 \mathrm{~K}$, there is a Curie-Weiss temperature dependence due to the presence of some paramagnetic impurities.

The magnetization $M(H)$ of the $\mathrm{V}_{0.98}{ }^{57} \mathrm{Fe}_{0.02}$ alloy at $1.8 \mathrm{~K}$ versus applied fields is presented in Fig. $2 \mathrm{~b}$. Negative values of $M(H)$ obtained for $H<5 \mathrm{kOe}$ can be taken as demonstration of the Meissner effect in the studied material. Moreover, the $M(H)$ behaviour in low fields is typical for a type-II superconductor in the vortex state. According to the presented results, the upper critical field $H_{c 2}$ is close to $4.8 \mathrm{kOe}$, while the lower critical field $H_{c 1}$ is not higher than 100 Oe at $1.8 \mathrm{~K}$.

Selected recorded TMS spectra of $\mathrm{V}_{0.98}{ }^{57} \mathrm{Fe}_{0.02}$ are presented in Fig. 3. We observe only one doublet with small quadrupole splitting over the whole temperature range. Abnormal behaviour of the hyperfine parameters at or near $T_{\mathrm{c}}$ is not observed, which contrasts to $\mathrm{FeSe}_{0.5} \mathrm{Te}_{0.5}$ exhibiting phonon softening below its $T_{\mathrm{c}}=12.5 \mathrm{~K}[7]$. At the same time, the complete absence of magnetic ordering rather indicates the paramagnetic character of $\mathrm{Fe}$ in the $\mathrm{V}_{0.98}{ }^{57} \mathrm{Fe}_{0.02}$ sample. This result is in agreement with previous Mössbauer data for $\mathrm{V}-\mathrm{Fe}$ alloys $[8,9]$ as well as by theoretical calculations [5].

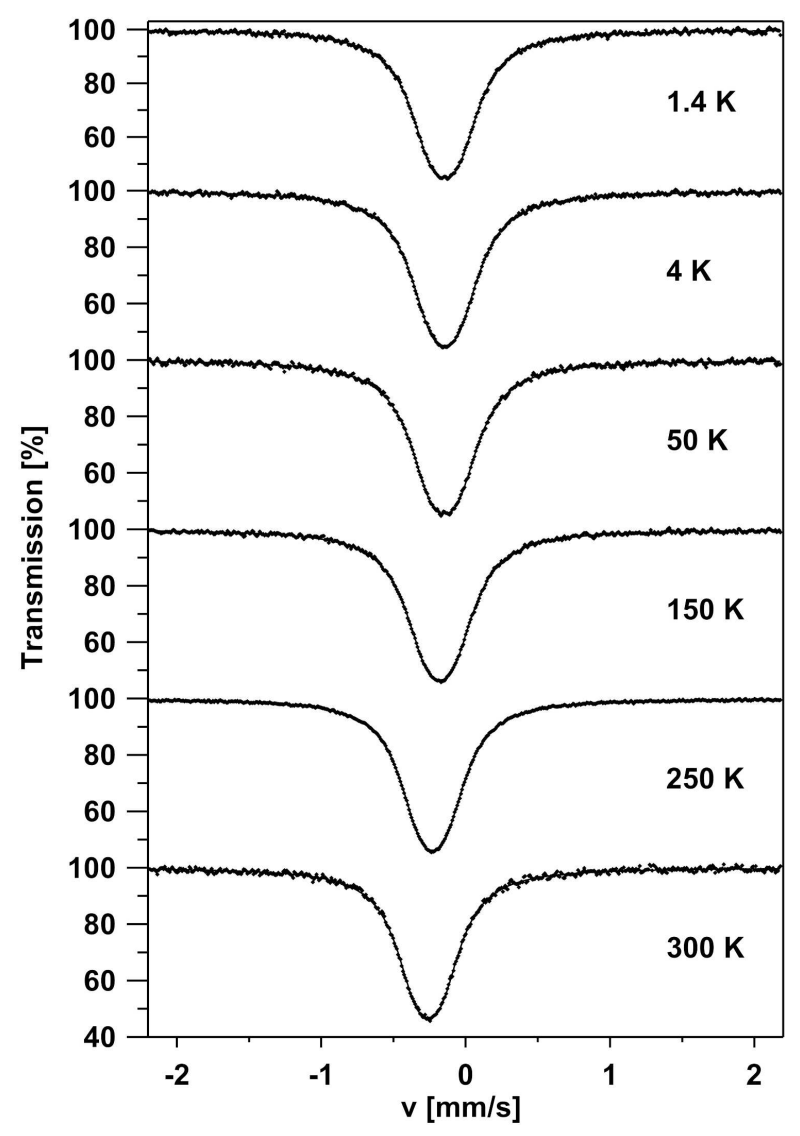

Fig. 3. TMS spectra of $\mathrm{V}_{0.98}{ }^{57} \mathrm{Fe}_{0.02}$ alloy in the temperature range $1.4 \mathrm{~K}-300 \mathrm{~K}$. The continuous line is a fitting; see text. 
The presence of small quadrupole splitting in measured spectra is probably connected with the random placement of $\mathrm{V}$ and Fe atoms at all bcc lattice sites which causes a departure from exact cubic symmetry about the ${ }^{57} \mathrm{Fe}$ atoms [8]. Figure 4 shows the temperature dependence of $Q S$ obtained from the fits of the TMS spectra. Similar temperature dependence of $Q S$ were observed in many metallic systems and it could be well described by the empirical equation $[10,11]$ :

$$
Q S(T)=Q S(0)\left(1-B T^{3 / 2}\right),
$$

where $Q S(0)$ is the value of $Q S$ at $0 \mathrm{~K}$ and $B$ is a constant. The fit of $Q S(T)$ experimental values to Eq. (1) gives $Q S(0)=0.170(1) \mathrm{mm} / \mathrm{s}$ and $B=1.4(2) \times$ $10^{-5} \mathrm{~K}^{-3 / 2}$. The obtained $B$ value is comparable to those found for other compounds. Note that the physical meaning of this fitting parameter is not fully understood.

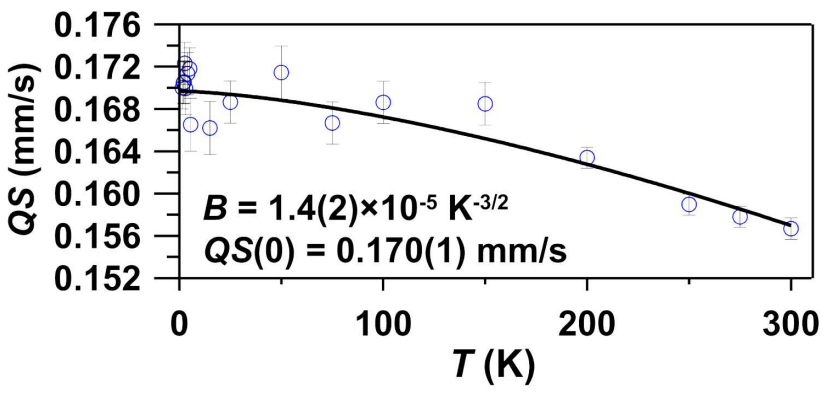

Fig. 4. Temperature dependence of $Q S$ obtained from the TMS spectra. The solid line represents the fit to Eq. (1).

The temperature dependence of $I S$ is presented in Fig. 5a. Clearly, the $I S$ values increase convexly with decrease of temperature, thus the isomer shift appears to vary with temperature as it was expected due to the second-order Doppler (SOD) effect. Taking into account that the second-order Doppler shift depends on the lattice vibrations of the Fe atoms, the $I S(T)$ dependence could be expressed in terms of the Debye model of the lattice vibrations as [12]:

$$
I S(T)=I S_{0}-\frac{9}{2} \frac{k_{\mathrm{B}} T}{M c}\left(\frac{T}{\Theta_{\mathrm{D}}}\right)^{3} \int_{0}^{\Theta_{\mathrm{D}} / T} \frac{x^{3} \mathrm{~d} x}{\mathrm{e}^{x}-1},
$$

where $I S_{0}$ is the temperature-independent isomer shift, $k_{\mathrm{B}}$ is the Boltzmann constant, $M$ is the mass of ${ }^{57} \mathrm{Fe}$, $c$ is the speed of light in vacuum and $\Theta_{\mathrm{D}}$ is the Debye temperature. Fitting experimental data to Eq. (2) yields $I S_{0}=0.037(1) \mathrm{mm} / \mathrm{s}$ and $\Theta_{\mathrm{D}}=511(11) \mathrm{K}$.

The Debye temperature could be determined alternatively using the temperature dependence of the Mössbauer spectral area $C$, which is proportional to the Lamb-Mössbauer factor $f$. The latter quantity represents the fraction of the recoil free transitions in comparison with the total number of transitions. According to the Debye theory, the temperature dependence of the spectral area could be described by [12]:

$$
\begin{aligned}
& C(T)= \\
& k \exp \left\{\frac{-3 E_{\gamma}^{2}}{M c^{2} k_{\mathrm{B}} \Theta_{\mathrm{D}}}\left[\frac{1}{4}+\left(\frac{T}{\Theta_{\mathrm{D}}}\right)^{2}\right] \int_{0}^{\Theta_{\mathrm{D}} / T} \frac{x \mathrm{~d} x}{\mathrm{e}^{x}-1}\right\},
\end{aligned}
$$

where $k$ is a proportionality constant and $E_{\gamma}$ is the energy of of the Mössbauer transition. The fit of the collected data (Fig. 5b) to Eq. (3) gives $\Theta_{\mathrm{D}}=692(10) \mathrm{K}$. This $\Theta_{\mathrm{D}}$ value is much higher than that estimated from the $I S(T)$ dependence. Such disagreement may be explained by assumption of two different approximations: (i) The average mean-square vibrational displacements of the atoms in the case of $C(T)$ and (ii) The mean-square velocity in the case of $I S(T)$. The weighted average of the above two $\Theta_{\mathrm{D}}$ values is $602(90) \mathrm{K}$.
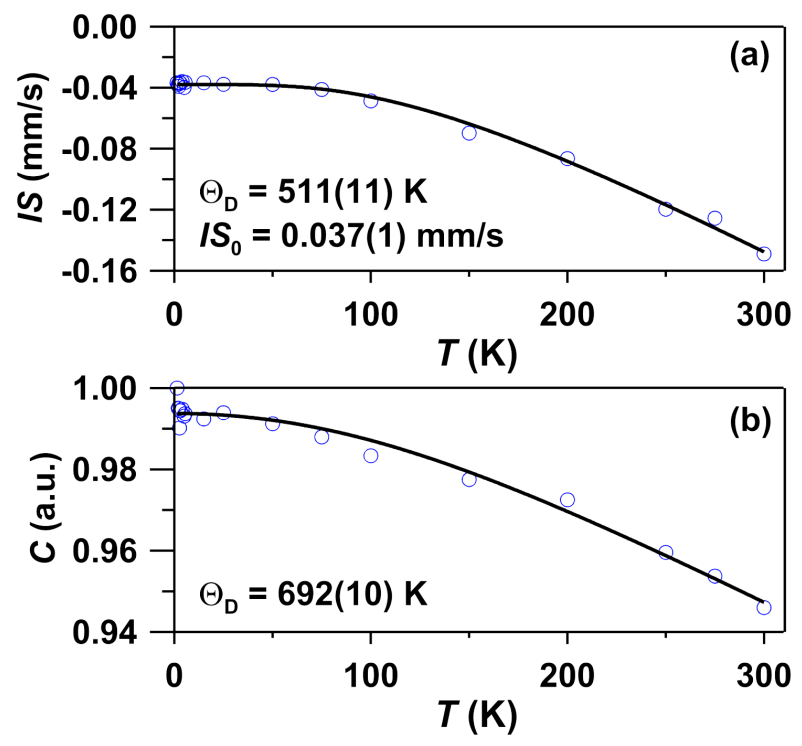

Fig. 5. Temperature dependence of (a) the $I S$ values and (b) the absorption spectral area $C$. The standard uncertainties for the presented quantities do not exceed 1\%. The solid lines represent the fits to Eq. (2) in (a) and to Eq. (3) in (b).

Using a relation

$$
m \Theta_{\mathrm{D}}^{2}=\sum \mu_{i} m_{i} \Theta_{\mathrm{D}, i}^{2}
$$

where $m$ and $\Theta_{\mathrm{D}}$ are molar mass and the Debye temperature of the alloy, respectively. The index $i=1$ and 2 denotes constituted atoms with respective fraction $\mu_{i}$, atomic mass $m_{i}$ and the Debye temperature $\Theta_{\mathrm{D}, i}$. Taking $\Theta_{\mathrm{D}, V}=399 \mathrm{~K}$ [13], the obtained for the $\mathrm{V}_{0.98}{ }^{57} \mathrm{Fe}_{0.02}$ alloy $\Theta_{\mathrm{D}}=404.6 \mathrm{~K}$. This value is somewhat larger than $\Theta_{\mathrm{D}}=383 \mathrm{~K}$ reported previously for $\mathrm{V}_{0.9811} \mathrm{Fe}_{0.0189}$ alloy by Isino et al. [4].

Finally, the determined values of $T_{\mathrm{c}}=3.7 \mathrm{~K}$ and $\Theta_{\mathrm{D}}=404.6 \mathrm{~K}$ were used to evaluate the electronphonon coupling constant $\bar{\lambda}_{\text {el-ph }}$ with the McMillan equation [14]:

$$
\bar{\lambda}_{\mathrm{el}-\mathrm{ph}}=\frac{1.04+\mu^{*} \ln \left(\frac{\Theta_{\mathrm{D}}}{1.45 T_{\mathrm{c}}}\right)}{\left(1-0.62 \mu^{*}\right) \ln \left(\frac{\Theta_{\mathrm{D}}}{1.45 T_{\mathrm{c}}}\right)-1.04},
$$


where $\mu^{*}$ is the Coulomb pseudo-potential parameter, which is assigned usually between 0.1 and 0.15 . Assuming $\mu^{*}=0.125$, the calculated $\bar{\lambda}_{\mathrm{el}-\mathrm{ph}}=0.54$ value indicates a weak electron-phonon coupling superconductivity in the $\mathrm{V}_{0.98}{ }^{57} \mathrm{Fe}_{0.02}$ alloy.

\section{Conclusions}

The results obtained by dc-magnetic measurements and TMS can be concluded as follows:

1. The $\mathrm{V}_{0.98}{ }^{57} \mathrm{Fe}_{0.02}$ alloy is a type-II conventional superconductor with $T_{\mathrm{c}} \approx 3.7 \mathrm{~K}$ and $H_{c 2}$ close to $4.8 \mathrm{kOe}$ at $1.8 \mathrm{~K}$.

2. The complete absence of magnetic ordering of ${ }^{57} \mathrm{Fe}$ is evidenced by the Mössbauer spectroscopy spectra and the paramagnetism of the studied sample.

3. There is no discernible feature at or near $T_{\mathrm{c}}$ in any of the hyperfine parameters. Once more, it was shown that a transition to the superconducting state is seldom observable by the Mössbauer spectroscopy, as the formation of the Cooper pairs and subsequent Bose condensate have no effect on the local hyperfine interactions in $\mathrm{V}_{0.98}{ }^{57} \mathrm{Fe}_{0.02}$.

4. The Debye temperature of the ${ }^{57} \mathrm{Fe}$ atoms $\Theta_{\mathrm{D}}=$ $602(90) \mathrm{K}$ determined directly from the Mössbauer measurements is served to estimate $\Theta_{\mathrm{D}}=404.6 \mathrm{~K}$ of the alloy.

5. The calculated $\bar{\lambda}_{\mathrm{el}-\mathrm{ph}}=0.54$ indicates a weak electron-phonon coupling superconductivity in the $\mathrm{V}_{0.98}{ }^{57} \mathrm{Fe}_{0.02}$ alloy.

\section{References}

[1] R. Radebaugh, P.H. Keesom, Phys. Rev. 149, 209 (1966).

[2] R. Radebaugh, P.H. Keesom, Phys. Rev. 149, 217 (1966).

[3] S.T. Sekula, R.H. Kernohan, Phys. Rev. B 5, 904 (1972).

[4] M. Isino, K. Akutsu, Y. Muto, J. Phys. Soc. Jpn. 54, 289 (1985).

[5] S. Mirbt, I.A. Abrikosov, B. Johansson, H.L. Skriver, Phys. Rev. B 55, 67 (1997).

[6] R. Idczak, R. Konieczny, J. Chojcan, J. Appl. Phys. 115, 103513 (2014).

[7] J. Lindén, J.-P. Libäck, M. Karppinen, E.-L. Rautama, H. Yamauchi, Solid State Commun. 151, 130 (2011).

[8] R.S. Preston, D.J. Lam, M.V. Nevitt, D.O. Van Ostenburg, C.W. Kimball, Phys. Rev. 149, 440 (1966).

[9] J.C. Krause, J. Schaf, M.I. da Costa, C. Paduani, Phys. Rev. B 61, 6196 (2000).

[10] Z.M. Stadnik, P. Wang, H.-D. Wang, C.-H. Dong, M.-H. Fang, J. Alloys Comp. 561, 82 (2013).

[11] M.A. Albedah, F. Nejadsattari, Z.M. Stadnik, J. Przewoźnik, J. Alloys Comp. 619, 839 (2015).

[12] Mössbauer Spectroscopy and Transition Metal Chemistry: Fundamentals and Applications, Eds. P. Gütlich, E. Bill, A. Trautwein, Springer, Berlin 2011.

[13] A. Tari, The Specific Heat of Matter at Low Temperatures, Imperial College Press, 2003.

[14] W.L. McMillan, Phys. Rev. 167, 331 (1968). 